\title{
El perfil de la mortalidad en el Estado de Yucatán, México.
}

\section{Artículo Original}

Gilberto Balam-Pereira, Ernesto Ochoa-Estrada, Genny Sonda-Ortiz.

Departamento de Ecología Humana, Centro de Investigaciones y Estudios Avanzados del Instituto Politécnico Nacional, Unidad Mérida, Mérida, Yucatán, México.

\section{RESUMEN.}

Introducción. Este trabajo tiene por objetivo conocer las principales causas de la mortalidad en el Estado de Yucatán, México y las diferencias que presentan sus distintas regiones económicas, a fin de que los resultados sean útiles para la planeación de los programas estatales de salud.

Material y método. Se revisaron las actas de defunción (1996-1998), en 76 municipios (71\%) de un total de 106 que componen el Estado, seleccionados por muestreo estratificado. Se usó también información de la Secretaría de Salud sobre las defunciones por desnutrición del período de 1990-1997. Se calcularon las tasas de acuerdo a la proyección de la población, en base a los datos del XI Censo de Población y Vivienda 1990 de INEGI.

Resultados. Se destaca que las causas crónicas degenerativas han cobrado relevancia en el medio rural a expensas de aquellas de origen infectocontagioso. La desnutrición es causa de defunción en todos los grupos de edad; la bronconeumonía, las enfermedades congénitas, y la asfixia neonatal predominan en la infancia; los accidentes son importantes en la edad escolar; las cardiopatías, las neoplasias, la cirrosis hepática y la diabetes figuran principalmente en los adultos.

Discusión. Los resultados obtenidos se confrontan con las estadísticas nacionales, se presentan las diferencias por regiones y se comenta la causalidad de los principales padecimientos en la infancia, así como las inconveniencias del subregistro de problemas importantes como el SIDA y el suicidio. (Rev Biomed 2002; 13:1-8)

Palabras clave: epidemiología, Yucatán México, cirrosis hepática, desnutrición.

\section{SUMMARY.}

\section{Mortality Profile of Yucatan, Mexico.}

Introduction. The aim at this study was to determine the principal causes of mortality within the State of Yucatan, Mexico and the differences

Solicitud de sobretiros: Dr. Gilberto Balam-Pereira, Cinvestav del Instituto Politécnico Nacional, Unidad Mérida, Km. 6, Antigua Carretera a Progreso. C.P. 97310, Mérida, Yucatán, México. 


\section{G Balam-Pereira, E Ochoa-Estrada, G Sonda-Ortiz.}

found among the different economical regions so that the results can be useful for the planning of the state health programs.

Material and methods. The material and method consisted in checking death certificates (1996$1998)$ in 76 municipalities $(71 \%)$ of the 106 which conform the state of Yucatán, Mexico. The municipalities were selected by stratified sampling. Data from the Secretary of Health about malnutrition was also used in several age groups (1990-1997). In order to project (annual values) population, data from the XI Census of Population and Housing 1990 by the National Institute of Statistics, Geography and Computer Science was used, as well as growing information by the Health Public Sector.

Results. It is noted that chronic-degenerative causes stand out within the rural area at the expense of those of an infect-contagious origin. Malnutrition is cause of death in all age groups mainly. Broncopneunia, congenital illnesses and cot death were predominant among infants. Accidents are important causes among school age children; cardiopathies, neoplasias, hepatic cirrosis and diabetes were the main causes among adults. Some cases were caused by stress and others by social factors suicide, and AIDS.

Discussion. The results obtained are compared to national statistics, differences between studied regions are also presented; causality of death in childhood is discussed; problems in relation to registers of AIDS and suicide are also commented in this paper. (Rev Biomed 2002; 13:1-8)

Key words: Epidemiology, Yucatan Mexico, liver cirrhosis, malnutrition.

\section{INTRODUCCIÓN.}

Existen muchas definiciones sobre epidemiología, pero una muy aceptable es la de Maxcy (1): "Rama de la medicina que estudia las relaciones entre los diversos factores y condiciones que determinan la frecuencia y

\section{Revista Biomédica}

distribución de un proceso infeccioso o estado fisiológico en una comunidad humana". Aunque Gordon (2) agrega un concepto interesante: la epidemiología es una ecología médica", o sea, el hombre y sus relaciones con el medio natural.

En otros trabajos $(3,4)$ hemos estudiado las principales determinantes que definen las regiones económicas, los indicadores de marginación, las variables con fuerza de cohesión que los agrupan en regiones críticas, las frecuencias y causas del fenómeno migratorio rural, la mortalidad, el problema de la desnutrición infantil y la fecundidad.

En el presente estudio abordamos una de las áreas fundamentales de la demografía que es la mortalidad, su magnitud y cambios en Yucatán, México en el periodo de 1996-1998.

\section{MATERIAL Y MÉTODO.}

Por muestreo estratificado (5) se seleccionaron 76 municipios representativos (71\%) de un total de 106 que componen el estado de Yucatán, México, considerando además las distintas regiones económicas definidas por el Consejo Nacional de Población (6). En estos municipios se revisaron las actas de defunción correspondientes al período de 1996-1998, registrándose la causa principal de cada fallecimiento, para después calcular las tasas de los mismos según la edad y género de los casos. Se usó también la información que nos proporcionó la Secretaría de Salud sobre las defunciones por desnutrición en diferentes grupos de edad correspondientes al período 1990-1997.

Para la proyección de la población nos basamos en los datos obtenidos del XI Censo de Población y Vivienda 1990 de INEGI y en el crecimiento poblacional manejado por el sector salud. 
Mortalidad en el Estado de Yucatán.

Cuadro 1

Mortalidad por grupos de edad y región. Yucatán 1996-1998

Tasas por mil nacidos vivos y por 10 mil habs.

\begin{tabular}{lcrrc}
\hline Región & $\begin{array}{c}<\mathbf{1} \text { año } \\
\text { por mil n.v. }\end{array}$ & $\mathbf{1}$ a $\mathbf{4}$ años & $\mathbf{5}$ a 14 años & de 15 y más \\
\hline Hortícola & 94.9 & 0.6 & 7.2 & 245.0 \\
Costera & 1.5 & 2.2 & 4.7 & 149.7 \\
Exhenequenera & 93.0 & 10.0 & 4.3 & 174.0 \\
Ganadera & 27.4 & 15.0 & 3.5 & 143.0 \\
Milpera & 20.0 & 5.6 & 0.8 & - \\
Metropolitana & 8.4 & 10.0 & 3.0 & 86.8 \\
\hline
\end{tabular}

Fuente: Encuesta Cinvestav 1996-1998. Registros Civiles Municipales.

\section{RESULTADOS.}

En el cuadro 1 se presenta la mortalidad general por grupos de edad y región. La neonatal, perinatal y por desnutrición, por región se presenta en los cuadros 2 y 3 .

En los cuadros 4 a 9 se observa las principales causas de defunción por región y grupos de edad.

Finalmente, en el cuadro 10 se presenta el total de defunciones por grupos de edad y causas.

Con referencia al SIDA, en la revisión de las libretas de defunción de los Registros Civiles de los municipios, encontramos en los tres últimos años 5 defunciones: 3 en la zona henequenera, 1 en la costera y 1 en la hortícola lo que arroja una baja tasa en relación a la población del Estado. La baja tasa aparente de SIDA como causa de defunción, registrados en las libretas del Registro Civil de los municipios. creemos que se debe a un registro deficiente o subregistro porque en la mayoría de los casos, se enmascara como causa de defunción. En 1999 Yucatán ocupó el quinto lugar a nivel nacional. Los servicios de salud habían registrado 900 casos. La edad más frecuente en que está presente este flagelo es entre los 15 y 44 años de edad. Mérida, Motul, Progreso, Valladolid y Tizimín son los municipios con mayor índice de enfermos. Por tipo de actividad, los más afectados son los empleados (217), profesionales
(120), trabajadores manuales (91), comerciantes (45) y amas de casa (37). De los 638 enfermos en 1997, 573 eran hombres (incluyendo 7 casos pediátricos) y 65 mujeres ( 12 casos pediátricos).

\section{DISCUSIÓN.}

Calculamos que en 1997, la población total de Yucatán, México, era de 1'631,304 habitantes, con una tasa de crecimiento anual de 2.4 por ciento. Hasta 1997, disponíamos de las diferencias de la mortalidad infantil y preescolar en tasas superiores a la nacional: en menores de un año, la nacional era de 16.4 por mil nacidos vivos y la de las regiones estudiadas 25.0; la

Cuadro 2

Mortalidad neonatal y perinatal en las regiones estudiadas, 1996-1998.

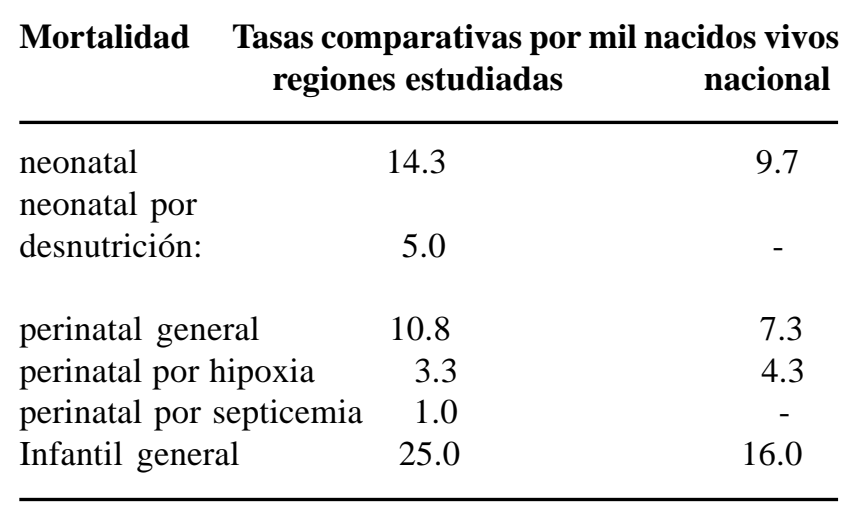

Fuente: Encuesta Cinvestav 1996.1998. Registros Civiles Municipales.

Vol. 13/No. 1/Enero-Marzo, 2002 
G Balam-Pereira, E Ochoa-Estrada, G Sonda-Ortiz.

Cuadro 3

Mortalidad por Desnutrición en Niños 0-14 años de edad.

Yucatán 1990 - 1997, Tasas por 100 mil habitantes, por grupo de edad y región.

\begin{tabular}{lcccc}
\hline Región & $<$ de 1 año & 1 a 4 años & 5 a 14 años & Total \\
\hline Costera & 11.6 & 3.4 & 2.7 & 5.9 \\
Ganadera & 46.0 & 2.4 & 0.4 & 16.2 \\
Henequenera & 48.6 & 9.4 & 0.8 & 19.6 \\
Hortofrutícola & 36.2 & 13.3 & 17.5 & 22.3 \\
Metropolitana & 110.0 & 11.6 & 6.9 & 42.8 \\
Maicera & 52.6 & 37.7 & 0.0 & 30.1 \\
\hline
\end{tabular}

Fuente: Datos de la SSA, Mérida, Yuc. 1997. Procesó CINVESTAV 1999.

nacional preescolar de 1 a 4 años fue de 1.1 y la estatal de 1.9. La mortalidad de la edad escolar del estado fue igual a la nacional, de 0.4 por mil.

De acuerdo a los datos del cuadro 1, sobresale la mortalidad infantil de la región hortícola y la exhenequenera. Se observa además que las regiones costera y metropolitana son las que tienen las menores tasas.

El cuadro 2 presenta como región metropolitana los municipios de Kanasín y Umán, donde la alta mortalidad por desnutrición se explica por la población marginada que ha emigrado hacia estos municipios en los que la situación socioeconómica es precaria. Umán multiplicó 3.5 veces su población y Kanasín lo hizo 5.5 tantos en el período 1990-1995. Es notoria la diferencia que mantiene este indicador en la zona costera.

Es de notarse que la desnutrición ocupa un lugar destacado como causa de defunción en todos los grupos de edad, es decir, desde el nacimiento hasta la edad senil. Tiene esta causa un primer lugar en los menores de cinco años, el tercer lugar en los escolares y el octavo en los

Cuadro 4

Tasas de mortalidad de la región hortícola. Yucatán 1996-1998.

\begin{tabular}{|c|c|c|c|c|}
\hline HORTICOLA & $\begin{array}{l}<1 \text { a ñ o } \\
\text { por mil }\end{array}$ & $\begin{array}{l}1-4 \text { a ñ o } \mathrm{s} \\
\text { por } 10 \text { mil }\end{array}$ & $\begin{array}{l}5-14 \text { a ñ o s } \\
\text { por } 100 \text { mil }\end{array}$ & $\begin{array}{l}15 \text { y más } \\
\text { por } 10 \text { mil }\end{array}$ \\
\hline desnutrición & 27.0 & 22.6 & 16.0 & 19.7 \\
\hline asfixia neonatal & 16.2 & - & - & - \\
\hline bronconeumonía & 13.1 & 13.1 & 8.0 & 22.6 \\
\hline congénita & 11.5 & 3.7 & - & - \\
\hline gastroenteritis & 9.2 & 5.6 & - & - \\
\hline septicemia & 6.1 & 5.6 & - & - \\
\hline accidentes & - & - & 16.0 & 13.5 \\
\hline cardiopatías & - & - & 16.0 & 46.0 \\
\hline neoplasias & - & - & 8.0 & 24.2 \\
\hline cáncer cerv. uterino & - & - & - & 7.0 \\
\hline meningitis & - & - & 8.0 & - \\
\hline accid. vascular cerebral & - & - & - & 23.8 \\
\hline diabetes & - & - & - & 22.6 \\
\hline cirrosis & - & - & - & 21.4 \\
\hline
\end{tabular}

Fuente: Encuesta Cinvestav 1996-1998. Registros Civiles Municipales.

\section{Revista Biomédica}


Mortalidad en el Estado de Yucatán.

Cuadro 5

Mortalidad de la región costera. Yucatán 1996-1998.

\begin{tabular}{lcccc}
\hline COSTERA & $\begin{array}{l}<\text { a ñ o } \\
\text { por mil }\end{array}$ & $\begin{array}{c}1-4 \text { a ñ o s } \\
\text { por 10 mil }\end{array}$ & $\begin{array}{c}5-14 \text { a ñ o s } \\
\text { por 100 mil }\end{array}$ & $\begin{array}{c}15 \text { y más } \\
\text { por 10 mil }\end{array}$ \\
\hline bronconeumonía & 7.5 & 74.7 & - & 14.8 \\
asfixia neonatal & 7.5 & - & - & - \\
congénita & 5.0 & 18.6 & - & - \\
accidentes & - & 37.3 & 37.8 & 11.7 \\
cardiopatías & - & 37.3 & - & 50.8 \\
gastroenteritis & - & 18.6 & - & - \\
desnutrición & - & 37.3 & 9.4 & 3.1 \\
accid.vascular cerebral & - & - & 9.4 & 10.5 \\
neoplasias & - & - & - & 19.1 \\
diabetes & - & - & - & 10.2 \\
cirrosis & - & - & - & 2.2 \\
suicidio & - & - & - & 2.8 \\
\hline
\end{tabular}

Fuente: Encuesta Cinvestav 1996.1998, Registros Civiles Municipales.

mayores de 14 .

Obsérvese en el cuadro 3 que la mortalidad neonatal del estudio, es $47.4 \%$, mayor que la nacional. La perinatal representa el $75.5 \%$ de la neonatal y el $43.2 \%$ de la infantil general de las regiones estudiadas. La neonatal es el $57.2 \%$ de la infantil también general. Por otra parte, encontramos que en la mortalidad neonatal (14.3) el $34.6 \%$ se debe a desnutrición.

La gravedad de estas tasas elevadas de mortalidad infantil no es otra cosa que el resultado de las deficiencias de los centros de salud en la atención del primer y segundo nivel, falta de continuidad de programas de control prenatal,

Cuadro 6

Mortalidad de la región henequenera. Yucatán. 1996-1998.

\begin{tabular}{|c|c|c|c|c|}
\hline HENEQUEN & $\begin{array}{l}<1 \text { a ñ o } \\
\text { por } 10 \text { mil }\end{array}$ & $\begin{array}{l}1-4 \text { a ñ o } \mathrm{s} \\
\text { por } 100 \mathrm{mil}\end{array}$ & $\begin{array}{c}5-14 \text { a ñ o s } \\
\text { por } 100 \text { mil }\end{array}$ & $\begin{array}{l}15 \text { y más } \\
\text { por } \mathbf{1 0} \text { mil }\end{array}$ \\
\hline desnutrición & 23.4 & 34.5 & - & 4.0 \\
\hline accidentes & 23.4 & 115 & 35.6 & 7.0 \\
\hline congénita & 23.4 & 13.7 & - & - \\
\hline bronconeumonía & 11.7 & 23.0 & 5.1 & 20.2 \\
\hline gastroenteritis & 11.7 & - & - & - \\
\hline septicemia & 5.9 & - & - & - \\
\hline neoplasia & - & 11.5 & - & 15.7 \\
\hline cáncer cerv. uterino & - & - & - & 5.0 \\
\hline cirrosis & - & - & - & 17.0 \\
\hline accid.vasc.cerebral & - & - & - & 16.0 \\
\hline diabetes & - & - & - & 15.3 \\
\hline infec. vías urinarias & - & - & 5.1 & 4.6 \\
\hline cardiopatías & - & 11.5 & - & 37.8 \\
\hline meningitis & - & - & 5.1 & - \\
\hline
\end{tabular}


G Balam-Pereira, E Ochoa-Estrada, G Sonda-Ortiz.

Cuadro 7

Mortalidad de la región ganadera. Yucatán. 1996-1998.

\begin{tabular}{|c|c|c|c|c|}
\hline GANADERA & $\begin{array}{l}<1 \text { a ñ o } \\
\text { por mil }\end{array}$ & $\begin{array}{l}1-4 \text { a ñ o s } \\
\text { por } 10 \text { mil }\end{array}$ & $\begin{array}{l}5-14 \text { a ñ o s } \\
\text { por } 100 \text { mil }\end{array}$ & $\begin{array}{l}15 \text { y más } \\
\text { por } \mathbf{1 0} \mathbf{~ m i l}\end{array}$ \\
\hline desnutrición & 10.2 & 6.4 & 4.4 & 18.0 \\
\hline bronconeumonía & 4.1 & 4.2 & - & 16.3 \\
\hline congénita & 3.7 & 1.1 & - & - \\
\hline gastroenteritis & 3.7 & 1.1 & 4.4 & - \\
\hline accidentes & 1.4 & 1.0 & 13.2 & 6.2 \\
\hline asfixia neonatal & 1.9 & - & - & - \\
\hline septicemia & 0.9 & 1.1 & - & - \\
\hline neoplasias & - & - & 4.4 & 10.3 \\
\hline cáncer cerv. uterino & - & - & - & 4.3 \\
\hline cardiopatías & - & - & 4.4 & 33.7 \\
\hline homicidio & - & - & 4.4 & - \\
\hline accid. vasc. cer. & - & - & - & 9.9 \\
\hline diabetes & - & - & - & 13.5 \\
\hline cirrosis & - & - & - & 11.4 \\
\hline
\end{tabular}

Fuente: Encuesta Cinvestav 1996-1998. Registros Civiles Municipales

capacitación de recursos humanos como parteras empíricas, personal médico y paramédico de primer y segundo nivel, así como la disponibilidad de recursos materiales de urgencia, como oxígeno, incubadoras, succionadores, medicamentos y materiales de curación.

Las enfermedades gastrointestinales y del aparato respiratorio que son controlables y prevenibles continúan figurando como causas importantes de defunción en los menores de un año.

El perfil de mortalidad en el grupo de 1 a 4 es similar al de menores de un año, con ligero aumento en las infecciones respiratorias.

En cuanto a la edad escolar, son de destacarse los accidentes, en su mayoría por causas de tránsito vehicular y las cardiopatías por causas congénitas y por fiebre reumática. En segundo lugar, figuran las leucemias en este grupo de 5 a 14 años.

Cuadro 8

Mortalidad de la región milpera. Yucatán 1996-1998.

\begin{tabular}{lcccc}
\hline MILPERA & $\begin{array}{c}<\text { a ñ o } \\
\text { por mil }\end{array}$ & $\begin{array}{c}1-4 \text { a ñ o s } \\
\text { por 10 mil }\end{array}$ & $\begin{array}{c}5-14 \text { a ñ o s } \\
\text { por 100 mil }\end{array}$ & $\begin{array}{c}15 \text { y más } \\
\text { por 10 mil }\end{array}$ \\
\hline septicemia & 12.2 & - & - & - \\
congénita & 12.2 & - & - & - \\
desnutrición & 4.0 & 2.3 & - & 7.30 \\
bronconeumonía & 2.4 & 5.7 & 0.8 & 2.2 \\
gastroenteritis & 2.0 & - & - & 1.1 \\
cardiopatías & - & - & - & 5.9 \\
neoplasias & - & - & - & 5.8 \\
accid. vasc cereb & - & - & 0.4 & - \\
accid vehicular & - & - & - & 4.4 \\
diabetes & - & & & -4 \\
\hline
\end{tabular}

Fuente: Encuesta Cinvestav 1996-1998. Registros Civiles Municipales

\section{Revista Biomédica}


Mortalidad en el Estado de Yucatán.

\section{Cuadro 9}

Mortalidad de la región metropolitana. Yucatán 1996-1998.

\begin{tabular}{lcccc}
\hline METROPOLIT & $\begin{array}{c}<\text { a ñ o } \\
\text { por mil }\end{array}$ & $\begin{array}{c}1-4 \text { a ñ o s } \\
\text { por 10 mil }\end{array}$ & $\begin{array}{c}5-14 \text { a ñ o s } \\
\text { por 10 mil }\end{array}$ & $\begin{array}{c}15 \text { y más } \\
\text { por 10 mil }\end{array}$ \\
\hline congénitas & 19.6 & 1.1 & - & - \\
gastroenteritis & 14.7 & 1.1 & - & 1.8 \\
bronconeumonía & 9.8 & 2.2 & - & 10.5 \\
desnutrición & 9.8 & 3.3 & - & - \\
asfixia & 9.8 & - & - & - \\
septicemia & 5.0 & - & - & 2.5 \\
accidentes & 5.0 & 1.1 & 1.0 & - \\
meningitis & 5.0 & - & 1.5 & 8.9 \\
neoplasias & - & - & 0.5 & 15.7 \\
cardiopatías & - & - & - & 8.0 \\
cirrosis & - & - & - & 6.4 \\
accid. vasc. cer. & - & - & - & 5.2 \\
diabetes & - & - & - & 2.7 \\
inf. v. urinarias & - & - & - & 3.5 \\
cáncer cerv. uterino & & & - & - \\
\hline
\end{tabular}

Fuente: Encuesta Cinvestav 1996-1998. Registros Civiles Municipales.

En los adultos predominan las causas propias infecciones del aparato respiratorio, la diabetes, la de esta edad, como son las enfermedades crónicas cirrosis hepática ( $4^{\circ}$ y $5^{\circ}$ lugares estas dos últimas) degenerativas, a las que hay que agregar las y la caquexia en los de edad avanzada. Merece

Cuadro 10

Mortalidad total de las regiones. Yucatán 1996-1998.

\begin{tabular}{lcccc}
\hline TOTAL & $\begin{array}{c}<1 \text { a ñ o } \\
\text { por mil }\end{array}$ & $\begin{array}{c}1-4 \text { a ñ o s } \\
\text { por 10 mil }\end{array}$ & $\begin{array}{c}5-14 \text { a ñ o s } \\
\text { por 100 mil }\end{array}$ & $\begin{array}{c}15 \text { y más } \\
\text { por 10 mil }\end{array}$ \\
\hline desnutrición & 7.3 & 6.9 & 5.0 & 8.5 \\
congénitas & 3.7 & 1.2 & - & - \\
asfixia & 3.2 & - & - & - \\
bronconeumonía & 3.0 & 5.0 & 3.0 & 17.6 \\
gastroenteritis & 2.4 & 1.5 & 0.7 & 2.0 \\
septicemia & 2.0 & 1.0 & - & - \\
accidentes & 1.2 & 1.2 & 16.0 & -0 \\
meningitis & 0.6 & - & 4.5 & 37.0 \\
cardiopatías & 0.5 & 0.8 & 5.0 & 16.0 \\
neoplasias & 0.1 & 0.2 & 6.0 & 2.8 \\
inf. vías urinarias & - & - & 1.0 & - \\
homicidio & - & - & 1.1 & 13.8 \\
accid. vasc. cer. & - & - & - & 13.9 \\
diabetes & - & - & - & 14.0 \\
cirrosis & - & - & - & 1.5 \\
T.B.pulonar & - & - & - & 1.0 \\
suicidio & - & - & - & \\
\hline
\end{tabular}

Fuente: Encuesta Cinvestav 1996-1998. Registros Civiles Municipales.

Vol. 13/No. 1/Enero-Marzo, 2002 


\section{G Balam-Pereira, E Ochoa-Estrada, G Sonda-Ortiz.}

especial mención el cáncer cérvico uterino, lo que obliga a intensificar su prevención como las demás neoplasias.

Volviendo a la cirrosis, este padecimiento afecta a más de 48 mil yucatecos en edad productiva -entre los 25 y 40 años- y hasta agosto de 1999 ya había causado en ese año 220 muertes en la entidad; aunque el alcohol es el principal determinante, un $20 \%$ se debe a algún tipo de hepatitis. Por su parte, la SSA, a través de su Sistema de Información Semanal de Vigilancia epidemiológica, destaca que Yucatán figura entre los diez estados con más casos de cirrosis hepática en 1999 (enero a agosto) después de Querétaro que figuró en noveno lugar, la entidad con mayor incidencia fue el Estado de México con 740 casos.

Con las defunciones a nivel nacional (7), hay coincidencias en los datos de Yucatán en lo que se refiere a la edad adulta: las cardiopatías, las neoplasias, la cirrosis y la diabetes son los principales flagelos. Pero también hay diferencias. Por ejemplo, los accidentes y muertes violentas constituyen la segunda causa de muerte en la población general del país. Esto no ocurre particularmente en Yucatán en donde sólo se produjo un caso de homicidio en edad escolar.

Sorpresivamente nos encontramos con un viejo enemigo que habíamos considerado como vencido. Este es, la tuberculosis pulmonar con una tasa de 15.0 por cien mil, que como se sabe está estrechamente relacionada con el mal estado nutricional y el deficiente sistema inmunológico. En 1994 hubo 112 casos y en 1998 se registraron 1999.

Con prestigio prehispánico, como causa común entre los yucatecos, sigue figurando el suicidio con una tasa importante de 10.0 por cien mil, ocupando el lugar No. 11 como motivo de deceso. En 1989 hicimos un primer estudio de este problema, en el que hubo 55 casos (8), y comparando con la situación actual, tenemos que: en el período 1997-1999 aumentó la frecuencia en un 62.3\%; en 1996 hubo 89 casos; en 1999, se registraron 105 y en el 2000 hubo 114. En la última década la tasa ha aumentado en más de un $50 \%$. En este año, fue más frecuente entre los 15 y 24 años y persisten los rasgos masculino, del medio urbano y los problemas económicos; la forma más común del suicidio es el ahorcamiento seguido por el uso de arma de fuego y tóxicos orales, según informes del Ministerio Público.

\section{AGRADECIMIENTO.}

El presente trabajo forma parte del Proyecto ConacytCinvestav, Ref. No. 26480-D, institución a la que expresamos nuestro agradecimiento por financiar el desarrollo del mismo.

\section{REFERENCIAS.}

1.- Maxcy KF. Preventive, Medicine and Hygiene. $7^{\mathrm{a}}$. Edition. Nueva York: Appleton- Century-Crofts Inc; 1956. p. 4.

2.- Gordon JE. The Newer Epidemiology. Tomorrow's Horizon in Public Health. En: Jacob HL. Nociones Prácticas de Epidemiología. Ediciones Científicas. México: La Prensa Médica Mexicana; 1984. p. 3-4.

3.- Balam G. Un fantasma recorre el mundo. El depredador más voraz de la historia. Mérida: Maldonado Editores; 1999. p. 126-36, 138-9,173-4.

4.- Balam G, Gurri F, Ochoa E, Sonda G. La zona maya centro de Yucatán. Estrategia de los campesinos ante los cambios. Mérida: Cinvestav Unidad; 1997. p. 20-36.

5.- Sheaffer R, Mendenhall W, Ott L. Elementos de Muestreo. México: Grupo Editorial Iberoamérica; 1987. p. $80-99$.

6.- Consejo Nacional de Población. Los cambios demográficos. México: Edic. CONAPO; 1988. p. 16-21

7.- V Informe Presidencial. México DF: 1999 (5 sep). La Jornada. Anexo Estadístico: p. 2-3

8.- Balam G. El suicidio en Yucatán. Revista Universidad Autónoma de Yucatán 1990; 5 (174):55-59.

\section{Revista Biomédica}

